\title{
Menstrual hygiene practice and its determinants among Adolescent Girls in Sub-Saharan Africa: A systematic review and meta-analysis protocol
}

Etsay Woldu Anbesu ( $\square$ etsaywold@gmail.com )

Samara University https://orcid.org/0000-0002-4532-6720

Setognal Birara Aychiluhm

Samara University

\section{Systematic Review}

Keywords: pooled prevalence, determinants, menstrual hygiene practice, adolescent girls, Sub-Saharan Africa

Posted Date: August 30th, 2021

DOI: https://doi.org/10.21203/rs.3.rs-640932/v3

License: (c) (i) This work is licensed under a Creative Commons Attribution 4.0 International License.

Read Full License 


\section{Abstract}

Background: Poor menstrual hygiene practices can lead to gynecological problems, psychosocial stress, and reduced access to school. Menstrual hygiene has not received adequate attention in Sub-Saharan Africa. Despite this, there are fragmented and inconsistent findings in Sub-Saharan Africa. Therefore, this systematic review and meta-analysis aimed to estimate the pooled prevalence of good menstrual hygiene practice and identify its associated factors among adolescent girls in Sub-Saharan Africa.

Methods: The protocol for this review was registered at PROSPERO with registration number: CRD42020165628. In this study, the Preferred Reporting Items for Systematic Reviews and MetaAnalyses guideline will be used to report the protocol. Online electronic databases PubMed, Google Scholar, CINAHL and unpublished grey literature will be searched to retrieve available studies from January 1-May/2021. The selection, data extraction, and quality assessment of studies will be carried by two authors. Joanna Briggs Institute checklist will be used to assess the quality of the studies. Heterogeneity among studies will be examined using a chi-squared test and I-squared statistic.

To investigate sources of heterogeneity, subgroup analyses and meta-regression will be done based on region and sample size. Sensitivity analysis will be conducted to identify influential studies. Publication bias will be examined by funnel plots and Egger's test. The statistical analysis will be conducted using STATA version-14 software. A random-effect model will be used to estimate the pooled prevalence, and statistical significance will be determined at a p-value of $<0.05$.

Discussion: Poor menstrual hygiene practice affects the health of millions of adolescent girls in developing countries. Currently, there are no synthesis research findings on the pooled prevalence of menstrual hygiene practice and its associated factor in Sub-Saharan Africa. Therefore, the findings of this systematic review and meta-analysis will be helpful to inform policy-makers, planners, and researchers to design appropriate interventions.

\section{Introduction}

Menstruation is a physiological process that occurs in most girls during puberty [1]. Good menstrual hygiene management ( $\mathrm{MHM}$ ) practice is described as "women and adolescent girls using a clean menstrual management material to absorb or collect blood that can be modified in privacy as often as necessary for the duration of the menstruation cycle, washing the body as needed with soap and water, and having access to facilities to dispose of used menstrual management materials" [2].

Globally, poor management of menstrual hygiene affects many school girls and women, particularly in developing nations [3, 4]. In low and middle-income countries, several girls cannot access or afford suitable sanitary materials and they use low-quality products such as new or old cloth, cotton wool, toilet paper, underwear alone, sponge, or not at all [5-9]. According to a United Nation International Children Emergency Fund (UNICEF) report, $10 \%$ of school-age African girls do not attend school during 
menstruation [10]. Moreover, a study done in five sub-Saharan African countries showed majority of adolescent girls reported a lack of safe, private, and clean toilets with washing facilities at schools [9].

Review literature showed that factors associated with poor menstrual hygiene practice were lack of access to clean and effective absorbents, facilities to change, disposal of absorbents, soap, and water in school settings, and lack of privacy $[3,5,11,12]$. Besides, consideration of menstruation as a taboo to discuss with others makes young girls limited knowledge about personal hygiene and develop fear and shame. Even adult women may not be aware of the biological factors of good hygienic practices, difficulties, and preventing them from seeking help [13-15].

Poor menstrual hygiene practices have many consequences. Expos adolescent girls and women to reproductive organ and urogenital infections, psychosocial stress, and reduced opportunities for accessing school and work [12, 16-18]. A study done in sub-Saharan African showed that about $49 \%$ of school girls missed four school days monthly due to menstruation [9]. Moreover, studies revealed many school girls suffer concentration, limited participation during class times due to discomfort and dishonor during menstruation [19-21].

In Sub-Saharan Africa countries, menstruation among school-age girls and women is a neglected issue. Adequate attention had not given by the water, sanitation, and hygiene (WASH), education sectors, sexual and reproductive health programs, despite the formal inclusion of menstrual hygiene under reproductive health [22]. Despite this, there were fragmented and inconsistent findings Sub-Saharan Africa. Thus, this systematic review and meta-analysis aimed to estimate the pooled prevalence of good menstrual hygiene practice and its associated factors among adolescent girls in Sub-Saharan Africa.

\section{Research question}

- What is the pooled prevalence of good menstrual hygiene practice among adolescent girls in SubSaharan Africa?

- What are the determinants of menstrual hygiene practice among adolescent girls in Sub-Saharan Africa?

\section{Objectives}

- To determine the pooled prevalence of good menstrual hygiene practice among adolescent girls in Sub-Saharan Africa.

- To identify the determinants of menstrual hygiene practice among adolescent girls in Sub-Saharan Africa.

\section{Methods}


The Preferred Reporting Items for Systematic review and Meta-analyses (PRISMA) was used to develop the review protocol [23], and PRISMA-P 2015 checklist [24] statements will be used to report the findings (Additional file 1). The protocol for this review had registered at PROSPERO with registration number: CRD42020165628

\section{PECO search guide}

Population: Adolescent girl (10-19 years) [25]

Exposure. Predictors or associated factors of menstrual hygiene practice. Factors are characteristics or exposures that increase or decrease the likelihood of menstrual hygiene practice among adolescent girls in Sub-Saharan Africa. Those factors include residence, age, maternal educational level, family income, menstrual flow duration, and knowledge on menses.

Comparison: This is the reported reference group for each associated factor in each study: adolescent girls residing in urban versus rural, from educated mothers versus no education, with good knowledge on menses versus poor knowledge.

Outcome. The primary outcome of the study will be the pooled prevalence of good menstrual hygiene practice. The second outcome of the study is associated factors of menstrual hygiene practice among adolescent girls in Sub-Saharan Africa. Good menstrual hygiene practice indicate when the studies report the overall good menstrual hygiene practice for the different measurement of menstrual hygiene practices (type of menstrual items used, maintenance of item if reusable or disposal if one-time use only, changing frequency, using a clean menstrual management material to absorb or collect, washing the body as needed with soap and water, access to facilities to dispose of used menstrual materials etc.).

\section{Searching strategy}

For the preparation and presentation, preferred Reporting Items for Systematic Reviews and MetaAnalysis guideline protocol will be used. Online electronic databases PubMed, Google Scholar, CINAHL and unpublished grey literature will be used to search articles from January 1-May/2021. The process of searching and selection of studies will be reported in accordance with the PRISMA diagram (Additional file 2). Cross-reference search will be done to add other related studies from the final included studies. Retrieve studies will be exported to Endnote version 8 reference manager software [26].

Medical Subject Heading (Mesh) text search term will be developed from articles PMID, author's keywords, titles and abstract. The search string will be developed using different Boolean operators, and modified, depending on the specific requirement of the database and relevant studies (Additional file 3). 


\section{Study selection and eligibility criteria}

First, duplicate studies will be removed from the Endnote citation manager. Two authors (EW and SB) will independently screen the studies based on inclusion and exclusion criteria. Irrelevant titles and articles will be excluded. However, relevant titles and articles with the full-text will be further screened. In case of articles which are not open access, we will contact the corresponding author. If the authors are not willing to provide full text, the articles will be excluded from the study. Studies that will be approved by both authors in the review processes will be included. During the review of the studies, any disagreement among reviewers will be resolved by discussion to reach a common understanding.

This review will include all observational studies: cross-sectional, analytical cross-sectional, case-control, and cohort studies. Articles published only with the English language, studies that report the overall good menstrual hygiene practice and its associated factors among adolescent girls will be included. However, study that only report overall good menstrual hygiene practice will also be included. If a studies analyzed knowledge, attitude and practice, we will only include the practice results. Institution and community based studies will be included. We will only consider the quantitative results for study that examine both quantitative and qualitative studies. Data that are difficult to extract will be excluded. Moreover, adolescent girls whose age group is not well defined, case reports, conference reports, national survey reports, and expert opinions will be excluded. No restriction will be made to the date of publication.

\section{Quality assessments}

Articles assessment using their title, abstract, and a full review of the manuscripts will be done before the inclusion of articles in the final meta-analysis. A critical appraisal will be performed by two authors (EW and SB) using the Joanna Briggs Institute Meta-Analysis of Statistics Assessment and Review Instrument (JBI-MASt-ARI) [27] (Additional file 4). Studies 50\% and above of the quality scale will be included and considered for systematic review and meta-analysis. For any scoring disagreements between the authors, the sources of discrepancy will be investigated through revision.

\section{Data extraction and management}

After identifying all eligible articles two independent reviewers (EW and SB) will extract the relevant data using an organized format on Microsoft Excel Spreadsheet 2016. Pretesting the data extraction form will be done before the beginning of the actual data extraction. If variations of extracted data exist, the phase will be repeated, and then discrepancies between data extractors will be discussed to reach a consensus. For each included article, we will record the author name, year of publication, the study area, study design, study period, sample size, the response rate, the target population, outcome definition, comparison 
groups, predictors, and overall good menstrual hygiene practice. For the practice studies, the prevalence, the logarithm of the prevalence, and standard error (SE) of the logarithm of the prevalence will be calculated. Similarly, for associated factors, OR, logarithms of OR, and SE of the logarithms of OR will be calculated. For any difficulties that might be encountered during data extraction, communication will be made with the corresponding author.

\section{Data synthesis and analysis}

The extract data will be imported into STATA version 14 software. A narrative description of the study population will be done, and tables and figures will be used to summarize the results.

A random-effect model will be used to estimate the pooled prevalence of good menstrual hygiene practice among adolescent girls in Sub-Saharan Africa [28]. The Freeman Tuckey variant of the arcsine square root transformation of proportions will be used to avoid variance variability when controlling proportions close to one [29, 30]. We will assess heterogeneity by using the chi-squared test on Cochran's $Q$ statistic with a $5 \%$ level of statistical significance [31] and $\mathrm{I}^{2}$ statistic test [32]. Assuming $\mathrm{I}^{2}$ values of $25 \%, 50 \%$, and $75 \%$ is representative of low, moderate, and high heterogeneity respectively. If the heterogeneity is significant $\left(\mathrm{I}^{2}>75 \%\right)$, and $\mathrm{p}$-value $<0.05$ will be declared as the presence of heterogeneity. Hence, subgroup analyses and meta-regression for continuous and categorical data based on region, and sample size will be done to investigate sources of heterogeneity at a $p$-value $<0.05$.

Sensitivity analysis will be done to see the effect of studies on the overall estimation on forest plots, and an individual study is suspected of excessive influence if the point estimate of its omitted analysis lies outside the confidence interval of the combined analysis or differs in significance relative to the combined analysis in forest plot. Publication bias will be examined by the visual inspection of funnel plots [33] and Egger's test [34]. A p-value $<0.05$ will be considered indicative of statistically significant publication bias. If evidence of publication bias present, the trim-and-fill (Duval and Tweedie's) method will be performed [35]. The statistical significance level will be declared at a $p$-value of less than 0.05 .

\section{Discussion}

This systematic review and meta-analysis protocol aim to synthesize research findings on the pooled prevalence of good menstrual hygiene practice and its associated factor in Sub-Saharan Africa. Poor menstrual hygiene practice affects the health of millions of adolescent girls, particularly in developing countries. Studies in developing countries have reported that more than $50 \%$ of girls have inadequate menstrual hygiene management (MHM) practices, with higher proportions in rural areas [36, 37]. Millions 
of adolescent girls suffer from complex problems, though good menstrual hygienic management practices can have many benefits [38].

A review of qualitative and quantitative research findings showed that poor menstrual hygiene practice affects school absenteeism, distraction, and detachment, poor psychosocial consequences, feelings of shame, fear of stigma, and anxiety [39-42]. Moreover, most young age women don't have comfort to discuss menses, as it is has a social taboo and could not have access to gain adequate information [43]. It is the overlooked opportunity to address the level of menstrual hygiene practice among girls earlier from their adolescent age in most low-income countries [44]. Thus, effective menstrual hygiene management practice is essential for women and girls to participate in society with dignity and comfort [4]. Consequently, it remains important to assess the pooled prevalence of good menstrual hygiene practice and its associated factors in Sub-Saharan Africa to inform the development of appropriate programs and policies that will have an impact in reducing poor menstrual hygiene practice in the region.

The finding from this systematic review will help national and non-governmental organizations in the health and education sector of each country of the region to emphasize the main factors that drive poor menstrual hygiene practice. Moreover, the finding will contribute to increase accessibility and availability of services to improve the health status of adolescent girls. Therefore, estimating the pooled prevalence of good menstrual hygiene practice and its associated factors in Sub-Saharan African using high-level evidence will be used to inform policy-makers, health programmers, education sectors, clinicians, decision making, and researchers.

This study protocol has the following limitation. Heterogeneity is common as we will consider varieties of study designs and from different geographic areas of Sub-Saharan African countries. Articles published other than English language will not be considered. Moreover, this protocol considers only observational studies design and excludes randomized clinical trials and quasi-experimental studies.

\section{Abbreviations}

JBI-MASt-ARI: Joanna Briggs Institute Meta-Analysis of Statistics Assessment and Review Instrument; PRISMA: Preferred Reporting Items for Systematic review and Meta-analyses; Mesh: Medical Subject Heading; MHM: Menstrual hygiene Management; SE: Standard error; UNICEF: United Nations International Children's Emergency Fund; WASH: Water, sanitation, and hygiene

\section{Declarations}

\section{Acknowledgements}

We would like to thank Samara University for the provision of free HINARY databases website and internet access 


\section{Author contribution}

EW and SB conceived and designed the systematic review and meta-analysis. EW drafted the protocol manuscript. EW and SB extensively reviewed and incorporated inputs in the protocol manuscript development. EW and SB read and approved the final version of the protocol.

\section{Funding}

No founder

\section{Availability of data and materials}

The systematic review and Meta-analysis protocol was submitted with additional files as supplementary materials.

Ethics approval and consent to participate

N/A

Consent for publication

Not applicable.

\section{Competing interests}

The authors declare that they have no competing interests.

\section{References}

1. Winkler, I.T. and V. Roaf, Taking the bloody linen out of the closet: menstrual hygiene as a priority for achieving gender equality. Cardozo JL \& Gender, 2014. 21: p. 1.

2. Biran, A., et al., Background paper on measuring WASH and food hygiene practices: definition of goals to be tackled post 2015 by the joint monitoring programme. London Sch Hyg Trop Med, 2012. 
2012: p. 81.

3. Sommer, M., et al., A time for global action: addressing girls' menstrual hygiene management needs in schools. PLoS medicine, 2016. 13(2): p. e1001962.

4. Sommer, M. and M. Sahin, Overcoming the taboo: advancing the global agenda for menstrual hygiene management for schoolgirls. American journal of public health, 2013. 103(9): p. 1556-1559.

5. Enzler, D.M., Knowledge, attitudes and practices concerning Menstrual Hygiene Management (MHM) of adolescents in rural primary schools in Malawi. 2019.

6. Fisseha, M.A., Y. Kebede, and H.Y. Yeshita, Menstrual hygiene practice and associated factors among secondary school girls in Wegera District, Northwest Ethiopia; a cross-sectional study. Computational Biology and Bioinformatics, 2017. 5(1): p. 6-11.

7. Geertz, A., An opportunity to address menstrual health and gender equity. 2016: FSG.

8. Kuhlmann, A.S., K. Henry, and L.L. Wall, Menstrual hygiene management in resource-poor countries. Obstetrical \& gynecological survey, 2017. 72(6): p. 356.

9. Tamiru, S., et al., Towards a sustainable solution for school menstrual hygiene management: cases of Ethiopia, Uganda, South-Sudan, Tanzania, and Zimbabwe. Waterlines, 2015: p. 92-102.

10. Education, P., Menstrual Hygiene Management-UNESCO Digital Library. United Nations Educational, Scientific and Cultural Organization: Paris, France, 2014.

11. Mason, L., et al., 'We keep it secret so no one should know'-A qualitative study to explore young schoolgirls attitudes and experiences with menstruation in rural Western Kenya. PloS one, 2013. 8(11): p. e79132.

12. Phillips-Howard, P.A., et al., Menstrual hygiene management among adolescent schoolgirls in low-and middle-income countries: research priorities. Global health action, 2016. 9(1): p. 33032.

13. Chothe, V., et al., Students' perceptions and doubts about menstruation in developing countries: a case study from India. Health promotion practice, 2014. 15(3): p. 319-326.

14. House, S., T. Mahon, and S. Cavill, Menstrual hygiene matters: a resource for improving menstrual hygiene around the world. Reproductive Health Matters, 2013. 21(41): p. 257-259.

15. Sommer, M., C. Sutherland, and V. Chandra-Mouli, Putting menarche and girls into the global population health agenda. Reproductive health, 2015. 12(1): p. 1-3.

16. Baker, K., et al., Menstrual Hygiene Practices, WASH Access and the Risk of Urogenital Infection in Women from Odisha, India: Supporting Information. 2015. 
17. Hulland, K.R., et al., Sanitation, stress, and life stage: a systematic data collection study among women in Odisha, India. PloS one, 2015. 10(11): p. e0141883.

18. Mathiyalagen, P., et al., A descriptive cross-sectional study on menstrual hygiene and perceived reproductive morbidity among adolescent girls in a union territory, India. Journal of family medicine and primary care, 2017. 6(2): p. 360.

19. Boosey, R., G. Prestwich, and T. Deave, Menstrual hygiene management amongst schoolgirls in the Rukungiri district of Uganda and the impact on their education: a cross-sectional study. The Pan African Medical Journal, 2014. 19.

20. Hennegan, J., et al., Measuring the prevalence and impact of poor menstrual hygiene management: a quantitative survey of schoolgirls in rural Uganda. BMJ open, 2016. 6(12).

21. Tegegne, T.K. and M.M. Sisay, Menstrual hygiene management and school absenteeism among female adolescent students in Northeast Ethiopia. BMC public health, 2014. 14(1): p. 1-14.

22. Ssewanyana, D. and B.K.Y. Bitanihirwe, Menstrual hygiene management among adolescent girls in sub-Saharan Africa. Global health promotion, 2019. 26(1): p. 105-108.

23. Moher, D., et al., Preferred reporting items for systematic reviews and meta-analyses: the PRISMA statement. PLoS medicine, 2009. 6(7): p. e1000097.

24. Shamseer, L., et al., Preferred reporting items for systematic review and meta-analysis protocols (PRISMA-P) 2015: elaboration and explanation. Bmj, 2015. 349.

25. Nation, U., United Nations Department of Economic and Social Affairs (UNDESA) 2007.

26. Bramer, W. and P. Bain, Updating search strategies for systematic reviews using EndNote. Journal of the Medical Library Association: JMLA, 2017. 105(3): p. 285.

27. Munn, Z., C. Tufanaru, and E. Aromataris, JBI's systematic reviews: data extraction and synthesis. AJN The American Journal of Nursing, 2014. 114(7): p. 49-54.

28. Berkey, C.S., et al., A random-effects regression model for meta-analysis. Statistics in medicine, 1995. 14(4): p. 395-411.

29. Lin, L. and C. Xu, Arcsine-based transformations for meta-analysis of proportions: Pros, cons, and alternatives. Health Science Reports, 2020. 3(3): p. e178.

30. Nyaga, V.N., M. Arbyn, and M. Aerts, Metaprop: a Stata command to perform meta-analysis of binomial data. Archives of Public Health, 2014. 72(1): p. 1-10.

31. Cooper, H., L.V. Hedges, and J.C. Valentine, The handbook of research synthesis and metaanalysis. 2019: Russell Sage Foundation. 
32. Higgins, J.P. and S.G. Thompson, Quantifying heterogeneity in a meta-analysis. Statistics in medicine, 2002. 21(11): p. 1539-1558.

33. Liu, J.L., The role of the funnel plot in detecting publication and related biases in meta-analysis. Evidence-based dentistry, 2011. 12(4): p. 121-122.

34. Egger, M., et al., Bias in meta-analysis detected by a simple, graphical test. Bmj, 1997. 315(7109): p. 629-634.

35. Duval, S. and R. Tweedie, Trim and fill: a simple funnel-plot-based method of testing and adjusting for publication bias in meta-analysis. Biometrics, 2000. 56(2): p. 455-463.

36. Adinma, E.D. and J. Adinma, Perceptions and practices on menstruation amongst Nigerian secondary school girls. African journal of reproductive health, 2008. 12(1): p. 74-83.

37. Bairwa, M., M. Rajput, and S. Sachdeva, Modified Kuppuswamy's socioeconomic scale: social researcher should include updated income criteria, 2012. Indian journal of community medicine: official publication of Indian Association of Preventive \& Social Medicine, 2013. 38(3): p. 185.

38. Nair, M., et al., Menstrual disorders and menstrual hygiene practices in higher secondary school girls. The Indian Journal of Pediatrics, 2012. 79(1): p. 74-78.

39. Crichton, J., et al., Emotional and psychosocial aspects of menstrual poverty in resource-poor settings: a qualitative study of the experiences of adolescent girls in an informal settlement in Nairobi. Health care for women international, 2013. 34(10): p. 891-916.

40. Grant, M., C. Lloyd, and B. Mensch, Menstruation and school absenteeism: evidence from rural Malawi. Comparative education review, 2013. 57(2): p. 260-284.

41. Lloyd, C.B. and J. Young, New lessons: The power of educating adolescent girls $-A$ girls count report on adolescent girls. 2009.

42. Sommer, M., et al., A comparison of the menstruation and education experiences of girls in Tanzania, Ghana, Cambodia and Ethiopia. Compare: A Journal of Comparative and International Education, 2015. 45(4): p. 589-609.

43. Wall, L.L., et al., A community-based study of menstrual beliefs in Tigray, Ethiopia. International Journal of Gynecology \& Obstetrics, 2016. 135(3): p. 310-313.

44. Chandra-Mouli, V. and S.V. Patel, Mapping the knowledge and understanding of menarche, menstrual hygiene and menstrual health among adolescent girls in low-and middle-income countries. Reproductive health, 2017. 14(1): p. 1-16.

\section{Supplementary Files}


This is a list of supplementary files associated with this preprint. Click to download.

- Additionalfile1.doc

- Additionalfile2.docx

- Additionalfile3.docx

- Additionalfile4.docx 